\title{
DiffPy-CMI - a software toolbox for structure analysis by Complex Modeling method.
}

\author{
$\underline{\text { P. Juhás }}^{1}$ and S. J. L. Billinge $\mathrm{e}^{2,3}$ \\ ${ }^{1}$ Computational Science Initiative, Brookhaven National Laboratory, Upton, New York 11973, USA \\ ${ }^{2}$ Condensed Matter Physics and Materials Science Department, \\ Brookhaven National Laboratory, Upton, New York 11973, USA \\ ${ }^{3}$ Department of Applied Physics and Applied Mathematics, \\ Columbia University, New York, New York 10027, USA
}

In general the feasibility and difficulty of structure solution from diffraction experiments arises from a balance between the complexity of the studied structure, and the amount and quality of signal in experimental data. For simple crystal structures this balance is much in a favor of successful solution. Due to their periodicity and other symmetries the atom positions in crystals can be all described with a few variables. At the same time their powder patterns collapse to a well-resolved, signal-rich Bragg reflections. This leads to an over-constrained structure determination problem with tractable solution. The situation is reversed for nanomaterials and systems with significant local distortions. Such structures are of lower symmetry and require more degrees of freedom for their characterization. Experimental data from such materials however tend to be more noisy, possess broad diffuse intensities, and overall carry insufficient signal for a unique identification of structure with more unknowns. One way of addressing this imbalance is to use Complex Modeling method. Complex Modeling incorporates multiple experimental and/or theoretical inputs and combines them in a common structure optimization routine. Complex Modeling requires a flexible and extensible software that permits to build multi-probe models tailored for the experimental and theoretical knowledge available for the studied material. I will present the DiffPy-CMI software ${ }^{1}$, which was purpose developed for such modeling, together with its several applications for structure analysis problems. The examples will include determination of the core structure in the $\mathrm{Au}_{144}(\mathrm{SH})_{60}$ nanoclusters $^{2}$, size and shape determination of quantized-growth CdSe nanoparticles from PDF and SAS measurements and PDF-derived solution of packing and orientation of molecules in organic crystals. ${ }^{3}$ The presentation will also discuss the uniqueness of obtained solutions and assess how is it affected by weighing of the utilized structure probes.

${ }^{1}$ P. Juhás, C. L. Farrow, X. Yang, K. R. Knox, and S. J. L. Billinge, Acta Crystallogr. A 71, 562 (2015).

2 K. M. Ø. Jensen, P. Juhas, M. A. Tofanelli, C. L. Heinecke, G. Vaughan, C. J. Ackerson, and S. J. L. Billinge, Nat. Commun. 7, 11859 (2016).

${ }^{3}$ D. Prill, P. Juhás, S. J. L. Billinge, and M. U. Schmidt, Acta Crystallogr. A 72, 62 (2016). 